\title{
The Compelling Issue of Nonvitamin K Antagonist Oral Anticoagulant Adherence in Atrial Fibrillation Patients: A Systematic Need for New Strategies
}

\author{
Marco Proietti ${ }^{1,2,3}$ Deirdre A. Lane ${ }^{3}$ \\ ${ }^{1}$ Department of Clinical Sciences and Community Health, University \\ of Milan, Milan, Italy \\ ${ }^{2}$ Geriatric Unit, Fondazione IRCCS Ca' Granda, Ospedale Maggiore \\ Policlinico, Milan, Italy \\ ${ }^{3}$ Liverpool Centre for Cardiovascular Science, University of Liverpool \\ and Liverpool Heart and Chest Hospital, Liverpool, \\ United Kingdom
}

Thromb Haemost 2020;120:369-371.

Address for correspondence Marco Proietti, MD, PhD, FESC, FEHRA, Geriatric Unit, Fondazione IRCCS Ca' Granda, Ospedale Maggiore Policlinico, Via Pace 9, 20122 Milan, Italy (e-mail: marco.proietti@unimi.it).

cohort of real-life AF patients enrolled in a single Korean tertiary referral cardiology department. ${ }^{7}$ They evaluated the adherence to treatment, expressed as percentage of prescribed doses taken (PDT), and also evaluated adherence with the Morisky Medication Adherence Scale (MMAS)-8 tool. In a cohort of $719 \mathrm{AF}$ patients prescribed one of the four NOACs (apixaban $47.8 \%$, dabigatran $21.2 \%$, rivaroxaban $18.4 \%$, and edoxaban $12.6 \%$ ), they found that over a mean (standard deviation [SD]) treatment period of 7.2 (5.7) months, the mean (SD) PDT for the once-daily NOACs (rivaroxaban and edoxaban) was $95.4(9.1 \%)$ and $93.4 \%(12.7 \%)$ for the twice-daily NOACs (dabigatran and apixaban). Overall, $92.2 \%$ of patients reported high adherence (PDT $\geq 80 \%$ ). Among the various NOACs, use of dabigatran was associated with the lowest adherence (PDT $=89.8 \%$ ), while in general the twice-daily dosing was associated with an increased risk of reporting poorer adherence (PDT $<80 \%$ ) in the univariate analysis (odds ratio [OR]: $2.15 ; 95 \%$ confidence interval [CI]: 1.06-4.34). A sensitivity analysis performed excluding dabigatran users found that twice-daily dosing no longer affected adherence. $^{7}$

The MMAS-8 was a good predictor of poor adherence, showing an AUC of $0.751(p<0.001)$, with a MMAS-8 $\geq 3$ exhibiting a $63.8 \%$ sensitivity and $78.5 \%$ specificity for poor adherence. In a multivariate logistic regression analysis, the twice-daily NOAC regimen was independently associated with a MMAS score of $\geq 3$ (OR: $1.90 ; 95 \% \mathrm{CI}: 1.35-2.67$ ). ${ }^{7}$

The data presented appear reassuring in terms of good adherence with NOAC in this cohort, with less than $10 \%$ of patients reporting a PDT $<80 \%$. However, the study does have some limitations, namely one Korean center only, relatively

(c) 2020 Georg Thieme Verlag KG Stuttgart · New York
DOI https://doi.org/ 10.1055/s-0040-1702231. ISSN 0340-6245. 


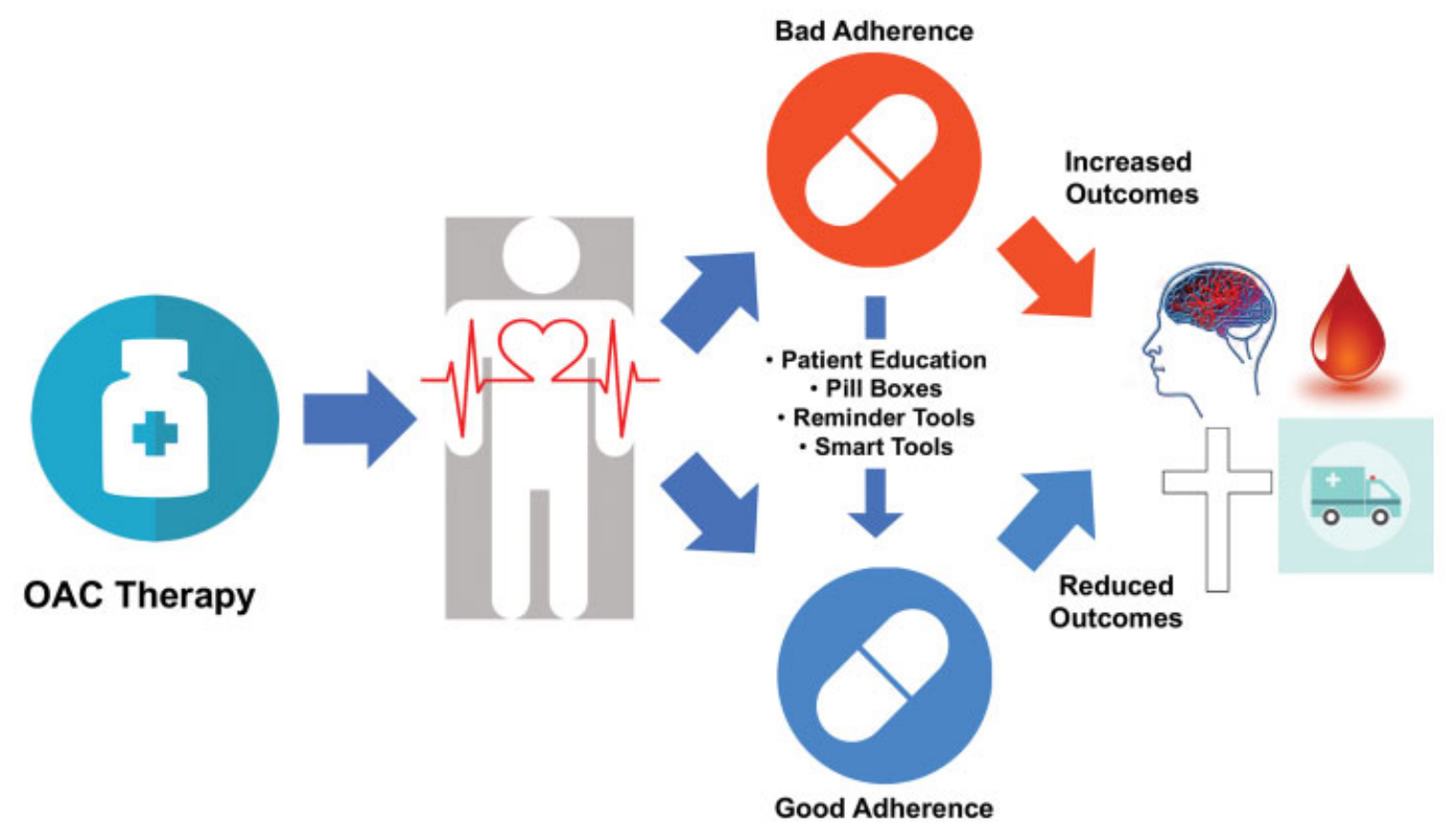

Fig. 1 Relationship between oral anticoagulant treatment, adherence, and outcomes in atrial fibrillation patients.

small cohort managed exclusively in a tertiary center with a limited follow-up period, which may impact the generalizability of the results.

A recent study performed among the UK primary electronic health records system (The Health Information Network), reports more concerning figures regarding OAC adherence. In this analysis, good adherence was defined as the proportion of days covered (PDC) of $>80 \%$. Good adherence among users of oral anticoagulant drugs was $55.2 \%$ overall, being lowest in VKA users (51.2\%) and significantly higher in NOACs users (dabigatran $66.5 \%$, rivaroxaban $63.1 \%$, and apixaban $64.7 \%)^{8}$ but still far from optimal. This study also showed that the rate of good adherence was lower in those patients with a shorter follow-up available. Previous data were similar indicating that over time the adherence rate was progressively lower, irrespective of the type of NOACs used. ${ }^{6}$

The paper by Hwang et al addresses an important issue regarding OAC management, that of adherence. Indeed, the ability of MMAS- 8 to predict the occurrence of a poor adherence is useful in terms of clinical management of these patients and could be utilized alongside other tools to evaluate AF patients. Indeed, the SAMe- $\mathrm{TT}_{2} \mathrm{R}_{2}$ score has been designed to identify those AF patients that would more likely perform well if prescribed with VKA, ${ }^{9-11}$ which is relevant since VKAs are still widely used OAC globally.

Despite all international guidelines currently recommending the use of NOACs over VKA for the majority of AF patients, ${ }^{12,13}$ optimal management of these patients should evaluate the most appropriate oral anticoagulant as part of an integrated care approach for AF patients. ${ }^{14-16}$ During the baseline evaluation of AF patients, use of MMAS- 8 could help to identify those patients that more likely will have a poor adherence to treatment and could be used to plan specific interventions to improve adherence.

Many factors are implicated in adherence to oral anticoagulant therapy among the patient-related factors are demographics, medical-related, behavioral factors, and patient understanding. ${ }^{6}$ Although many strategies to address nonadherence have been proposed, ${ }^{6}$ these need to be individually tailored to the patient based on the personal underlying cause (s) of non-adherence. Improving adherence to OAC in AF patients should be a priority of the clinical management of AF since data indicate that patients more adherent to NOACs are more likely to have better outcomes. ${ }^{17}$ Starting anticoagulation is not enough, we need to ensure that patients are adherent lifelong by asking about medication adherence and where non-adherence is identified, working with the patient to develop strategies to improve adherence and ensuring these are implemented and maintained (-Fig. $\mathbf{1}$ ).

\section{Conflict of Interest}

D.A.L. reports grants from Bristol-Myers-Squibb and Boehringer Ingelheim (paid to the institution), and personal fees from Boehringer Ingelheim, Bristol-MyersSquibb/Pfizer, Bayer, and Daichii-Sankyo, outside the submitted work. M.P. reports consultancy activity for Boehringer Ingelheim.

\section{References}

1 Connolly SJ, Ezekowitz MD, Yusuf S, et al; RE-LY Steering Committee and Investigators. Dabigatran versus warfarin in patients with atrial fibrillation. N Engl J Med 2009;361(12):1139-1151

2 Hohnloser SH, Basic E, Nabauer M. Changes in oral anticoagulation therapy over one year in 51,000 atrial fibrillation patients at 
risk for stroke: a practice-derived study. Thromb Haemost 2019; 119(06):882-893

3 Lip G, Freedman B, De Caterina R, Potpara TS. Stroke prevention in atrial fibrillation: Past, present and future. Comparing the guidelines and practical decision-making. Thromb Haemost 2017;117 (07):1230-1239

4 Wan Y, Heneghan C, Perera R, et al. Anticoagulation control and prediction of adverse events in patients with atrial fibrillation: a systematic review. Circ Cardiovasc Qual Outcomes 2008;1(02):84-91

5 Pritchett RV, Bem D, Turner GM, et al. Improving the prescription of oral anticoagulants in atrial fibrillation: a systematic review. Thromb Haemost 2019;119(02):294-307

6 Raparelli V, Proietti M, Cangemi R, Lip GY, Lane DA, Basili S. Adherence to oral anticoagulant therapy in patients with atrial fibrillation. Focus on non-vitamin $\mathrm{K}$ antagonist oral anticoagulants. Thromb Haemost 2017;117(02):209-218

7 Hwang J, Han S, Bae H-J, et al. NOAC adherence of patients with atrial fibrillation in the real world: dosing frequency matters? Thromb Haemost 2020;120(02):306-313

8 Banerjee A, Benedetto V, Gichuru P, et al. Adherence and persistence to direct oral anticoagulants in atrial fibrillation: a population-based study. Heart 2020;106(02):119-126

9 Zulkifly H, Lip GYH, Lane DA. Use of the SAMe-TT 2 R 2 score to predict anticoagulation control in atrial fibrillation and venous thromboembolism patients receiving vitamin $\mathrm{K}$ antagonists: a review. Hear Rhythm 2018;15(04):615-623

10 Proietti M, Lane DA, Lip GY. Relation of the SAMe-TT2R2 score to quality of anticoagulation control and thromboembolic events in atrial fibrillation patients: Observations from the SPORTIF trials. Int J Cardiol 2016;216(216):168-172

11 Proietti M, Lip GYH. Simple decision-making between a vitamin K antagonist and a non-vitamin $\mathrm{K}$ antagonist oral anticoagulant: using the SAMe-TT2R2 score. Eur Hear J Cardiovasc Pharmacother 2015;1(03):150-152

12 Proietti M, Lane DA, Boriani G, et al. Stroke prevention, evaluation of bleeding risk, and anticoagulant treatment management in atrial fibrillation contemporary international guidelines. Can J Cardiol 2019;35(05):619-633

13 Lip GYH, Banerjee A, Boriani G, et al. antithrombotic therapy for atrial fibrillation: CHEST guideline and expert panel report. Chest 2018;154(05):1121-1201

14 Lip GYH. The ABC pathway: an integrated approach to improve AF management. Nat Rev Cardiol 2017;14(11):627-628

15 Proietti M, Mujovic N, Potpara TS. Optimizing stroke and bleeding risk assessment in patients with atrial fibrillation: a balance of evidence, practicality and precision. Thromb Haemost 2018;118 (12):2014-2017

16 Yoon M, Yang P-S, Jang E, et al. Improved population-based clinical outcomes of patients with atrial fibrillation by compliance with the simple ABC (atrial fibrillation better care) pathway for integrated care management: a nationwide cohort study. Thromb Haemost 2019;119(10):1695-1703

17 Shehab A, Bhagavathula AS, Abebe TB, et al. Patient adherence to novel oral anticoagulants (NOACs) for the treatment of atrial fibrillation and occurrence of associated bleeding events: a systematic review and meta-analysis. Curr Vasc Pharmacol 2019;17(04):341-349 